\title{
Efficiency Loss of UE-CN Mixed Equilibrium Traffic Assignment under Road Pricing
}

\author{
Yu Xiao-Jun, Wang Ting-Ting \\ School of Mathematics and Statistics, \\ Guizhou University of Finance and Economics, \\ Guiyang, Guizhou, China \\ xjyu-myu@163.com
}

\begin{abstract}
The efficiency loss of two kinds of player (UE player and $\mathrm{CN}$ players) traffic assignment under road pricing is investigated, the users under UE player aim to minimize her/his own travel cost, the users belonging to the same $\mathrm{CN}$ player can fully cooperate with each other and different players will fully compete with each other, The users of one CN player aim to minimize their own total travel cost while compete with users of other players. Firstly, a variational inequality (VI) model is established to describing this mixed routing game. Then, the upper bound of UE$\mathrm{CN}$ mixed equilibrium traffic assignment is derived by analytic derivation when the road pricing is consider as (not consider as) part of total travel costs, respectively. Results show that the upper bound is a constant when road pricing is considered as part of total travel costs and the link travel cost functions satisfy some given conditions. The upper bound depends on the class of link travel cost functions and the road pricing scheme when road pricing is not considered as part of total travel costs.
\end{abstract}

KEYWORDS-UE-CN mixed equilibrium; efficiency loss; variational inequality; road pricing

\section{INTRODUCTION}

The Brass Paradox in traffic assignment shown that the user equilibrium (UE) is generally not identical with the system optimum (SO) as viewed from total travel cost [1]. However, the gap between the SO and the UE in the worst case had been unknown for a long time. Roughgarden and Tardos [2] introduced the efficiency loss (the price of anarchy) into traffic network and used it to quantify the difference between the UE and the SO, they achieved that for separable linear link travel cost functions, the upper bound of the efficiency loss is tight at 4/3. Karakostas and Kolliopoulos [3] showed it is $5 / 4$ after levying road pricing and road pricing were considered as part of the system cost. Reference [4] investigated the efficiency loss in fixed demand and elastic demand with road pricing scheme. Han and Yang [5] obtained several bounds of the efficiency loss for traffic equilibrium problem where there are multiple classes of users with a discrete set of value of time when the road pricing were considered (not considered) as part of the total travel cost, with the time-based criterion and the cost-based criterion, respectively. Later, researchers have studied the efficiency loss of transportation network under road pricing in different aspects [5-9].

Traffic assignment under UE principle is a nonatomic routing game by game terminology. It is a non-cooperative
Nash game with many infinitesimal users, and all users are competitive each other. In the case of SO, it is assumed that all users are cooperative and instructed by a single player to minimize the total cost of the system. This means there exist many infinitesimal players in the UE principle and there is only one central player in the SO principle. Harker [10] examined that the network has different CN players and UE player at the same time and obtained a new network equilibrium model. Reference [11] considered such a situation that the network has an additional player aiming to improve the overall system performance by controlling part of flows and further extended the above model. Yang and Zhang [12] studied the existence of anonymous link tolls in network with UE-CN mixed equilibrium behaviors. Yu and Huang [13] investigated the efficiency loss of UE-CN mixed equilibrium with polynomial cost functions by two approaches.

In this paper, we study the efficiency loss of UE-CN mixed equilibrium under road pricing. We suppose that all users following the UE principle in their routing decisions are considered as one single UE player and every $\mathrm{CN}$ player controls a strictly positive splittable flow. Under this assumption, in section 2, we present a VI formulation the traffic model of UE-CN mixed traffic assignment under road pricing. In section 3, we obtain the efficiency loss where road pricing are considered as part of total travel cost. In section 4, we investigate the efficiency loss where road pricing are not considered as part of total travel cost. In section 5 , we provide some concluding remarks.

\section{MODEL OF UE-CN MIXED EQUILIBRIUM TRAFFIC ASSIGNMENT UNDER ROAD PRICING}

Let $G=(N, A)$ be a directed transportation network, where $N$ and $A$ denote the sets of nodes and links, respectively. $U$ represents the UE player in the network. Let $K$ be the set of CN players in the network; $W^{U}$ be the set of Origin-Destination (OD) pairs where users obey UE principle; $W^{k}$ be the set of OD pairs where users are controlled by a CN player $k \in K$. Define $W^{K} \equiv \bigcup_{k \in K} W^{k}, W \equiv W^{U} \cup W^{K}$. Let $d_{w}$ be the demand between OD pair $w \in W ; R_{w}$ be the set of paths connecting OD pair $w \in W, R^{U} \equiv \cup_{w \in W^{U}} R_{w}, R^{k} \equiv$ $\cup_{w \in W^{k}} R_{w}, k \in K$. Suppose $f_{r}$ be the flow on path $r ; \delta_{a r}^{w}=1$ if the path $r \in R_{w}$ traverses link $a \in A$, and $\delta_{a r}^{w}=0$ otherwise; 
$v_{a}^{U}$ is the UE flow on link $a ; \mathbf{v}^{U} \equiv\left(\cdots, v_{a-1}^{U}, v_{a}^{U}, v_{a+1}^{U}, \cdots\right)$ is the vector of link flows by UE player; $v_{a}^{k}$ is the CN flow on link $a ; \mathbf{v}_{a} \equiv\left(v_{a}^{U}, v_{a}^{1}, \cdots, v_{a}^{k}, \cdots\right)$ is the vector of all flows on link $a$; $\mathbf{v}^{k} \equiv\left(\cdots, v_{a-1}^{k}, v_{a}^{k}, v_{a+1}^{k}, \cdots\right)$ is the vector of all link flows by CN player $k ; v_{a}^{K}=\sum_{k \in K} v_{a}^{k}$ is the total flow by all CN players on link $a ; \mathbf{v}^{K} \equiv\left(\cdots, \mathbf{v}^{k-1}, \mathbf{v}^{k}, \mathbf{v}^{k+1}, \cdots\right) ; v_{a}=v_{a}^{U}+v_{a}^{K}$ is the total flow on link $a$. Define $\mathbf{v} \equiv\left(\mathbf{v}^{U}, \mathbf{v}^{K}\right)$. Let $t_{a}\left(v_{a}\right)$ denote the average cost of traversing link $a \in A$. This cost function is assumed to be differentiable, convex, and monotonically increasing with the amount of link flow.

Suppose all OD demands are fixed. Thus, the feasible sets of link flows by the UE player and the CN players can be defined as follows:

$$
\begin{aligned}
& \Omega^{U}=\left\{\begin{array}{l}
\mathbf{v}^{U} \mid v_{a}^{U}=\sum_{r \in R^{U}} f_{r} \delta_{a r}^{w}, \quad \forall a \in A ; \\
\sum_{r \in R_{w}} f_{r}=d_{w} ; f_{r} \geq 0, r \in R^{U}, \forall w \in W^{U}
\end{array}\right\} \\
& \Omega^{k}=\left\{\begin{array}{l}
\mathbf{v}^{k} \mid v_{a}^{k}=\sum_{r \in R^{k}} f_{r} \delta_{a r}^{w}, \quad \forall a \in A ; \\
\sum_{r \in R_{w}} f_{r}=d_{w} ; f_{r} \geq 0, r \in R^{k}, \forall w \in W^{k}
\end{array}\right\}, k \in K
\end{aligned}
$$

Let $\Omega=\Omega^{U} \times \prod_{k \in K} \Omega^{k}, c_{a}^{U}, c_{a}^{k}$ be the travel cost of UE player and $\mathrm{CN}$ player on link $a$, respectively.

By the Lemma 1 in [13], we obtained the UE-CN mixed equilibrium traffic assignment under road pricing as following VI:

Lemma 1 Suppose $t_{a}\left(v_{a}\right), a \in A$ is monotonically increasing and convex, the vector $\mathbf{v}^{*} \in \Omega$ is the UE-CN mixed equilibrium under road pricing scheme $\boldsymbol{\tau}$ if and only if the following inequality holds

$$
\sum_{a \in A}\left\{\tilde{c}_{a}^{U}\left(\overline{\mathbf{v}}^{*}\right)\left(v_{a}^{U}-\bar{v}_{a}^{U^{*}}\right)+\sum_{k \in K} \tilde{c}_{a}^{k}\left(\overline{\mathbf{v}}^{*}\right)\left(v_{a}^{k}-\bar{v}_{a}^{k^{*}}\right)\right\} \geq 0, \quad \forall \mathbf{v} \in \Omega
$$

where $\tilde{c}_{a}^{U}(\mathbf{v})=t_{a}\left(v_{a}\right)+\tau_{a}, \tilde{c}_{a}^{k}(\mathbf{v})=t_{a}\left(v_{a}\right)+v_{a}^{k} t_{a}^{\prime}\left(v_{a}\right)+\tau_{a}$.

Since $t_{a}\left(v_{a}\right)$ is monotonically increasing and convex function, the mapping function $\tilde{\mathbf{c}}(\mathbf{v})=\left[\tilde{c}_{a}^{U}(\mathbf{v}), \tilde{c}_{a}^{k}(\mathbf{v}), \forall k\right]$ is continues with the total link flows. It is easy to know that $\Omega$ is non empty, closed and convex set, and then the VI problem (1) has at least one solution [14]. It is obvious that the VI problem (1) has unique solution if $\tilde{\mathbf{c}}(\mathbf{v})$ is strictly monotone function.

The SO model when road pricing not consider as part of system costs as following:

$$
\min _{\mathbf{v} \in \Omega} T(\mathbf{v})=\sum_{a \in A} t_{a}\left(v_{a}\right) v_{a}
$$

The SO model when road pricing consider as part of system costs as following:

$$
\min _{\mathbf{v} \in \Omega} \tilde{T}(\mathbf{v})=\sum_{a \in A}\left(t_{a}\left(v_{a}\right)+\tau_{a}\right) v_{a}
$$

If $t_{a}\left(v_{a}\right)$ is monotonically increasing and convex function, the optimization problem (2) and (3) has a unique solution in terms of the aggregate link flows. Let $\widehat{\mathbf{v}}$ and $\tilde{\mathbf{v}}$ be the solution of optimization problem (2) and (3), then the total travel costs of UE-CN mixed equilibrium at system optimum as following:

$$
\begin{array}{r}
T(\widehat{\mathbf{v}})=\sum_{a \in A} t_{a}\left(\widehat{v}_{a}\right) \hat{v}_{a}^{U}+\sum_{a \in A} \sum_{k \in K} t_{a}\left(\widehat{v}_{a}\right) \hat{v}_{a}^{k} \\
\tilde{T}(\tilde{\mathbf{v}})=\sum_{a \in A} t_{a}\left(\tilde{v}_{a}\right) \tilde{v}_{a}^{U}+\sum_{a \in A} \sum_{k \in K} t_{a}\left(\tilde{v}_{a}\right) \tilde{v}_{a}^{k}+\sum_{a \in A} \tau_{a} \tilde{v}_{a}
\end{array}
$$

Let $\overline{\mathbf{v}}$ be a solution of the minimization problem (1). Then the efficiency loss of the UE-CN mixed equilibrium when road pricing are not (are) part of system cost can be defined as $\rho=T(\overline{\mathbf{v}}) / T(\widehat{\mathbf{v}}), \tilde{\rho}=\tilde{T}(\overline{\mathbf{v}}) / \tilde{T}(\tilde{\mathbf{v}})$.It is easy to know $\rho, \tilde{\rho} \geq 1$. Next we focus on finding the upper bound of $\rho, \tilde{\rho}$.

\section{BOUNDS OF EFFICIENCY LOSS: ROAD PRICING IS PART OF TOTAL TRAVEL COSTS}

For $\overline{\mathbf{v}}$ be a solution of the minimization problem (1) and $\tilde{\mathbf{v}} \in \Omega$, with $\mathbf{v}$ replace by $\tilde{\mathbf{v}}$ in (1), we have

$$
\begin{aligned}
& \tilde{T}(\overline{\mathbf{v}}) \leq \tilde{T}(\tilde{\mathbf{v}})+\sum_{a \in A}\left(t_{a}\left(\bar{v}_{a}\right)-t_{a}\left(\tilde{v}_{a}\right)\right) \tilde{v}_{a}+\sum_{a \in A} \sum_{k \in K} \bar{v}_{a}^{k} t_{a}^{\prime}\left(\bar{v}_{a}\right)\left(\tilde{v}_{a}^{k}-\bar{v}_{a}^{k}\right) \\
& \leq \tilde{T}(\tilde{\mathbf{v}})+\max _{\mathbf{v} \in \Omega}\left\{\sum_{a \in A}\left(t_{a}\left(\bar{v}_{a}\right)-t_{a}\left(v_{a}\right)\right) v_{a}+\sum_{a \in A} t_{a}^{\prime}\left(\bar{v}_{a}\right) \sum_{k \in K} \bar{v}_{a}^{k}\left(v_{a}^{k}-\bar{v}_{a}^{k}\right)\right\} \\
& \leq \tilde{T}(\tilde{\mathbf{v}})+\max _{\mathbf{v} \geq 0}\left\{\sum_{a \in A}\left(t_{a}\left(\bar{v}_{a}\right)-t_{a}\left(v_{a}\right)\right) v_{a}+\sum_{a \in A} t_{a}^{\prime}\left(\bar{v}_{a}\right) \sum_{k \in K} \bar{v}_{a}^{k}\left(v_{a}^{k}-\bar{v}_{a}^{k}\right)\right\}
\end{aligned}
$$

Consider the following inequality:

$$
\sum_{k \in K} \bar{v}_{a}^{k}\left(v_{a}^{k}-\bar{v}_{a}^{k}\right) \leq \sum_{k \in K} \frac{1}{4}\left(v_{a}^{k}\right)^{2} \leq \frac{1}{4}\left(\sum_{k \in K} v_{a}^{k}\right)^{2} \leq \frac{1}{4}\left(v_{a}\right)^{2}
$$

where the first, second and last inequalities are satisfied respectively due to $\left(\frac{1}{2} v_{a}^{k}-\bar{v}_{a}^{k}\right)^{2} \geq 0, \forall k \in K, v_{a}^{k} \geq 0$ and $v_{a}=$ $v_{a}^{U}+\sum_{k \in K} v_{a}^{k}, v_{a}^{U} \geq 0$. Since $t_{a}^{\prime}\left(\bar{v}_{a}\right) \geq 0$, inequality (6) is equivalent to

$$
\tilde{T}(\overline{\mathbf{v}}) \leq \tilde{T}(\tilde{\mathbf{v}})+\max _{\mathbf{v} \geq 0}\left\{\sum_{a \in A}\left(t_{a}\left(\bar{v}_{a}\right)-t_{a}\left(v_{a}\right)\right) v_{a}+\frac{1}{4} \sum_{a \in A} t_{a}^{\prime}\left(\bar{v}_{a}\right)\left(v_{a}\right)^{2}\right\} .
$$

Let $H\left(v_{a}\right)=\left(t_{a}\left(\bar{v}_{a}\right)-t_{a}\left(v_{a}\right)\right) v_{a}+\frac{1}{4} t_{a}^{\prime}\left(\bar{v}_{a}\right)\left(v_{a}\right)^{2}, v_{a} \in[0,+\infty)$. Obviously, $H\left(v_{a}\right)$ is continuous and differentiable in domain, then $H^{\prime}\left(v_{a}\right)=t_{a}\left(\bar{v}_{a}\right)-t_{a}\left(v_{a}\right)-v_{a} t_{a}^{\prime}\left(v_{a}\right)+\frac{1}{2} v_{a} t_{a}^{\prime}\left(\bar{v}_{a}\right)$. Since $t_{a}\left(v_{a}\right)$ is monotony increasing convex function, thus $t_{a}^{\prime}\left(v_{a}\right) \geq t_{a}^{\prime}\left(\bar{v}_{a}\right) \geq 0$, we have $H^{\prime}\left(v_{a}\right) \leq 0$ under the condition $v_{a} \geq \bar{v}_{a} \geq 0$. This means $H^{\prime}\left(v_{a}\right)$ decreases with the variable $v_{a} \in\left[\bar{v}_{a},+\infty\right)$. Thus, we conclude that the second term in the 
right hand side (RHS) of (8) has the maximum within $\left[0, \bar{v}_{a}\right]$.

Before studying the upper bound of $\tilde{\rho}$, we give the lemma as follows.

Lemma 2 Supposed the link travel cost function $t_{a}\left(v_{a}\right)$ is a continuously differentiable, convex, monotonically increasing function and satisfying $t_{a}(c x) \geq c t_{a}(x)$ for $\forall c \in[0,1]$. Then, for each $v_{a} \geq 0$ such that $v_{a} t_{a}^{\prime}\left(v_{a}\right) \leq t_{a}\left(v_{a}\right)$.

Proof: For equation (7) in [3], it is easy to obtain that for each $v_{a} \geq 0$ such that

$$
v_{a} t_{a}^{\prime}\left(v_{a} / 2\right) \leq \int_{0}^{v_{a}} t_{a}^{\prime}(x) \mathrm{d} x=t_{a}\left(v_{a}\right)-t_{a}(0) \leq t_{a}\left(v_{a}\right) .
$$

The second inequality in (9) always holds due to $t_{a}(0) \geq 0$. In view of for $\forall c \in[0,1]$, inequality $t(c x) \geq c t(x)$ is satisfied. Thus,

$$
t_{a}\left(v_{a}\right) \leq 2 t_{a}\left(v_{a} / 2\right) .
$$

Combining with (9) and (10), $v_{a} t_{a}^{\prime}\left(v_{a} / 2\right) \leq 2 t_{a}\left(v_{a} / 2\right)$ holds. For this, with $v_{a}$ substituting $v_{a} / 2$, it is easy to obtain $v_{a} t_{a}^{\prime}\left(v_{a}\right) \leq t_{a}\left(v_{a}\right)$.

Rewrite the inequality (8) as

$$
\begin{aligned}
& \tilde{T}(\overline{\mathbf{v}}) \leq \tilde{T}(\tilde{\mathbf{v}})+\max _{v_{a} \geq 0}\left\{\sum_{a \in A}\left(t_{a}\left(\bar{v}_{a}\right)-t_{a}\left(v_{a}\right)\right) v_{a}+\frac{1}{4} \sum_{a \in A} t_{a}^{\prime}\left(\bar{v}_{a}\right)\left(v_{a}\right)^{2}\right\} \\
& =\tilde{T}(\tilde{\mathbf{v}})+\max _{u \in[0,1]}\left\{\sum_{a \in A}\left(t_{a}\left(\bar{v}_{a}\right)-t_{a}\left(u \bar{v}_{a}\right)\right) u \bar{v}_{a}+\frac{1}{4} \sum_{a \in A} t_{a}^{\prime}\left(\bar{v}_{a}\right)\left(u \bar{v}_{a}\right)^{2}\right\} \\
& \leq \tilde{T}(\tilde{\mathbf{v}})+\max _{u \in[0,1]}\left\{\sum_{a \in A}\left(t_{a}\left(\bar{v}_{a}\right)-u_{a}\left(\left(\bar{v}_{a}\right)\right) u_{a}^{-} \sharp \frac{u^{2}}{4} \sum_{a \in A} t_{a}^{\prime}\left(\bar{v}_{a}\right)\left(\bar{v}_{a}\right)^{2}\right\}\right. \\
& \leq \tilde{T}(\tilde{\mathbf{v}})+\max _{u \in[0,1]}\left\{\sum_{a \in A}\left(u-u^{2}\right) t_{a}\left(\bar{v}_{a}\right) \bar{v}_{a}+\frac{u^{2}}{4} \sum_{a \in A} t_{a}\left(\bar{v}_{a}\right) \bar{v}_{a}\right\} \\
& =\tilde{T}(\tilde{\mathbf{v}})+\max _{u \in[0,1]}\left(u-\frac{3}{4} u^{2}\right) \sum_{a \in A} t_{a}\left(\bar{v}_{a}\right) \bar{v}_{a} \\
& \leq \tilde{T}(\tilde{\mathbf{v}})+\frac{1}{3} \tilde{T}(\overline{\mathbf{v}})
\end{aligned}
$$

The first equation is satisfied as $v_{a}=u \bar{v}_{a}, u \in[0,1]$. The second inequality is satisfied as $t(c x) \geq c t(x)$ for each $c \in[0,1]$. The third inequality is satisfied for lemma 2 . The last inequality is satisfied for $\max _{u \in[0,1]}\left(u-\frac{3}{4} u^{2}\right)=\frac{1}{3}$ and $\sum_{a \in A} t_{a}\left(\bar{v}_{a}\right) \bar{v}_{a} \leq \sum_{a \in A}\left(t_{a}\left(\bar{v}_{a}\right)+\tau_{a}\right) \bar{v}_{a}=\tilde{T}(\overline{\mathbf{v}})$. Thus we have the following theorem:

Theorem 1 For a given separable link travel cost function class $C$, each instance $t_{a}\left(v_{a}\right) \in C$ is a continuously differentiable, convex, monotonically increasing function and satisfying $t_{a}(c x) \geq c t_{a}(x)$ for $\forall c \in[0,1] \cdot \overline{\mathbf{v}}$ is a solution of the problem (1) and $\tilde{\mathbf{v}}$ is a solution of the problem (3). Then

$$
\tilde{\rho}=\tilde{T}(\overline{\mathbf{v}}) / \tilde{T}(\tilde{\mathbf{v}}) \leq \frac{3}{2}
$$

Proof: From equation (11), we have $\tilde{T}(\overline{\mathbf{v}}) \leq \tilde{T}(\tilde{\mathbf{v}})+\frac{1}{3} \tilde{T}(\overline{\mathbf{v}})$, then $\tilde{\rho}=\tilde{T}(\overline{\mathbf{v}}) / \tilde{T}(\tilde{\mathbf{v}}) \leq \frac{3}{2}$.

IV. BOUNDS OF EFFICIENCY LOSS: ROAD PRICING IS NOT PART OF TOTAL TRAVEL COSTS

For $\overline{\mathbf{v}}$ be a solution of the minimization problem (1) and $\widehat{\mathbf{v}} \in \Omega$, with $\mathbf{v}$ replace by $\widehat{\mathbf{v}}$ in (1), we have

$$
\begin{aligned}
T(\overline{\mathbf{v}}) \leq & \sum_{a \in A} t_{a}\left(\bar{v}_{a}\right) \widehat{v}_{a}^{U}+\sum_{a \in A} \sum_{k \in K} t_{a}\left(\bar{v}_{a}\right) \hat{v}_{a}^{k}+ \\
& +\sum_{a \in A} \sum_{k \in K} \bar{v}_{a}^{k} t_{a}^{\prime}\left(\bar{v}_{a}\right)\left(\widehat{v}_{a}^{k}-\bar{v}_{a}^{k}\right)+\sum_{a \in A} \tau_{a}\left(\widehat{v}_{a}-\bar{v}_{a}\right) \\
= & \sum_{a \in A} t_{a}\left(\bar{v}_{a}\right) \widehat{v}_{a}+\sum_{a \in A} \sum_{k \in K} \bar{v}_{a}^{k} t_{a}^{\prime}\left(\bar{v}_{a}\right)\left(\widehat{v}_{a}^{k}-\bar{v}_{a}^{k}\right)+\sum_{a \in A} \tau_{a}\left(\widehat{v}_{a}-\bar{v}_{a}\right) \\
= & T(\widehat{\mathbf{v}})+\sum_{a \in A}\left(t_{a}\left(\bar{v}_{a}\right)-t_{a}\left(\widehat{v}_{a}\right)\right) \widehat{v}_{a}+ \\
& +\sum_{a \in A} \sum_{k \in K} \bar{v}_{a}^{k} t_{a}^{\prime}\left(\bar{v}_{a}\right)\left(\widehat{v}_{a}^{k}-\bar{v}_{a}^{k}\right)+\sum_{a \in A} \tau_{a}\left(\widehat{v}_{a}-\bar{v}_{a}\right)
\end{aligned}
$$

If we can find an maximum for the sum of the second, third and fourth term in the RHS of (13), we then obtain the upper bound of $\rho$. Let

$$
\begin{aligned}
F(\mathbf{v})= & \sum_{a \in A}\left(t_{a}\left(\bar{v}_{a}\right)-t_{a}\left(v_{a}\right)\right) v_{a}+\sum_{a \in A} \tau_{a}\left(v_{a}-\bar{v}_{a}\right)+ \\
& +\sum_{a \in A} t_{a}^{\prime}\left(\bar{v}_{a}\right) \sum_{k \in K} \bar{v}_{a}^{k}\left(v_{a}^{k}-\bar{v}_{a}^{k}\right), \mathbf{v} \in \Omega
\end{aligned} .
$$

First, we proof function $F(\mathbf{v})$ has the maximum within $\mathbf{v} \in \Omega$. For the feasible set $\Omega$ is convex set, we only need proof $F(\mathbf{v})$ is concave function within $\mathbf{v} \in \Omega$. Since $t_{a}\left(v_{a}\right)$ is strictly increasing and convex, and then $v_{a} t_{a}\left(v_{a}\right)$ is convex. For $\forall c \in[0,1], \forall \mathbf{v}^{\mathrm{I}}, \mathbf{v}^{\mathrm{II}} \in \Omega$, we can get $c v_{a}^{\mathrm{I}} t_{a}\left(v_{a}^{\mathrm{I}}\right)+(1-c) v_{a}^{\mathrm{II}}$

$$
\begin{aligned}
& t_{a}\left(v_{a}^{\mathrm{II}}\right) \geq\left(c v_{a}^{\mathrm{I}}+(1-c) v_{a}^{\mathrm{II}}\right) t_{a}\left(c v_{a}^{\mathrm{I}}+(1-c) v_{a}^{\mathrm{II}}\right) \quad \text { Thus, } \\
& c F\left(\mathbf{v}^{\mathrm{I}}\right)+(1-c) F\left(\mathbf{v}^{\mathrm{II}}\right) \\
& =\sum_{a \in A} t_{a}\left(\bar{v}_{a}\right)\left(c v_{a}^{\mathrm{I}}+(1-c) v_{a}^{\mathrm{II}}\right)-\sum_{a \in A}\left[c v_{a}^{\mathrm{I}} t_{a}\left(v_{a}^{\mathrm{I}}\right)+(1-c) v_{a}^{\mathrm{II}} t_{a}\left(v_{a}^{\mathrm{II}}\right)\right] \\
& +\sum_{a \in A} t_{a}^{\prime}\left(\bar{v}_{a}\right) \sum_{k \in K} \bar{v}_{a}^{k}\left(c v_{a}^{\mathrm{I}, k}+(1-c) v_{a}^{\mathrm{II}, k}\right)-\sum_{a \in A} t_{a}^{\prime}\left(\bar{v}_{a}\right) \sum_{k \in K}\left(\bar{v}_{a}^{k}\right)^{2} \\
& +\sum_{a \in A} \tau_{a}\left(\left(c v_{a}^{\mathrm{I}}+(1-c) v_{a}^{\mathrm{II}}\right)-\bar{v}_{a}\right) \\
& \leq \sum_{a \in A} t_{a}\left(\bar{v}_{a}\right)\left(c v_{a}^{\mathrm{I}}+(1-c) v_{a}^{\mathrm{II}}\right)-\sum_{a \in A} t_{a}\left(c v_{a}^{\mathrm{I}}+(1-c) v_{a}^{\mathrm{II}}\right)\left(c v_{a}^{\mathrm{I}}+(1-c) v_{a}^{\mathrm{II}}\right) \\
& +\sum_{a \in A} t_{a}^{\prime}\left(\bar{v}_{a}\right) \sum_{k \in K} \bar{v}_{a}^{k}\left(c v_{a}^{\mathrm{I}, k}+(1-c) v_{a}^{\mathrm{II}, k}\right)-\sum_{a \in A} t_{a}^{\prime}\left(\bar{v}_{a}\right) \sum_{k \in K}\left(\bar{v}_{a}^{k}\right)^{2} \\
& +\sum_{a \in A} \tau_{a}\left(\left(c v_{a}^{\mathrm{I}}+(1-c) v_{a}^{\mathrm{II}}\right)-\bar{v}_{a}\right) \\
& \quad=F\left(c \mathbf{v}^{\mathrm{I}}+(1-c) \mathbf{v}^{\mathrm{II}}\right)
\end{aligned}
$$

This means function $F(\mathbf{v})$ is a concave function. 


$$
\begin{aligned}
& \text { We define } \\
& \gamma_{1}(C, \boldsymbol{\tau})=\max _{t_{a} \in C, a \in A_{1}} \gamma_{a}\left(t_{a}, \bar{v}_{a}, \tau_{a}\right) \\
& =\max _{v_{a}^{U} \geq 0, v_{a}^{k} \geq 0} \frac{\left(t_{a}\left(\bar{v}_{a}\right)-t_{a}\left(v_{a}\right)\right) \nu_{a}+\sum_{k \in K} \bar{v}_{a}^{k}\left(v_{a}^{k}-\bar{v}_{a}^{k}\right)+\tau_{a}\left(v_{a}-\bar{v}_{a}\right)}{t_{a}\left(\bar{v}_{a}\right) \bar{v}_{a}}
\end{aligned}
$$

where $A_{1}=\left\{a \in A: 0 \leq \tau_{a} \leq \bar{v}_{a} t_{a}^{\prime}\left(\bar{v}_{a}\right)\right\}$. Then define

$$
\begin{aligned}
& \gamma_{2}(C, \boldsymbol{\tau})=\max _{t_{a} \in C, a \in A_{2}} \gamma_{a}\left(t_{a}, \bar{v}_{a}, \tau_{a}\right) \\
& =\max _{v_{a}^{U} \geq 0, v_{a}^{k} \geq 0} \frac{\left(t_{a}\left(\bar{v}_{a}\right)-t_{a}\left(v_{a}\right)\right) v_{a}+\sum_{k \in K} \bar{v}_{a}^{k}\left(v_{a}^{k}-\bar{v}_{a}^{k}\right)+\tau_{a}\left(v_{a}-\bar{v}_{a}\right)}{t_{a}\left(v_{a}\right) v_{a}}
\end{aligned}
$$

where $A_{2}=\left\{a \in A: \tau_{a}>\bar{v}_{a} t_{a}^{\prime}\left(\bar{v}_{a}\right)\right\}, A_{1} \cup A_{2}=A$ and by convention $0 / 0=0$.

For a given separable link travel cost function class $C$, let $v_{a}=\widehat{v}_{a}$ in formula (16) and (17), then,

$$
\begin{aligned}
& \sum_{a \in A}\left(t_{a}\left(\bar{v}_{a}\right)-t_{a}\left(\widehat{v}_{a}\right)\right) \widehat{v}_{a}+\sum_{a \in A} t_{a}^{\prime}\left(\bar{v}_{a}\right) \sum_{k \in K} \bar{v}_{a}^{k}\left(\widehat{v}_{a}^{k}-\bar{v}_{a}^{k}\right)+\sum_{a \in A} \tau_{a}\left(\widehat{v}_{a}-\bar{v}_{a}\right) \\
& \quad \leq \gamma_{1}\left(C, \boldsymbol{\tau} \mathbf{y} \boldsymbol{t} \mathbf{v}^{-}\right)+\gamma_{2}(C,) T\left(^{-}\right)
\end{aligned}
$$

This lead to

Theorem2. For a given separable link travel cost function class $C$, each instance $t_{a}\left(v_{a}\right)$ is a monotonically increasing convex function, the vector $\overline{\mathbf{v}}$ is the UE-CN mixed equilibrium under road pricing scheme $\boldsymbol{\tau}$, the vector $\hat{\mathbf{v}}$ is the SO of problem (2). Then,

$$
\rho=\frac{T(\overline{\mathbf{v}} \boldsymbol{\phi}}{T(\overline{\mathbf{v}} \boldsymbol{t}} \leq \frac{1+\gamma_{2}(C,)}{1-\gamma_{1}(C,)} .
$$

Proof: From equation (14) and (18), we have $T(\overline{\mathbf{v}}) \leq$ $T\left(\hat{\mathbf{v}} \mathbf{v}_{\mathbf{v} \tau \mathbf{W}_{1}}(\boldsymbol{C}, \quad) T\left(^{-}\right)+\gamma_{2}(\boldsymbol{C}) T,\left(^{-}\right)\right.$, then (19) is obtained.

Theorem 2 states that when the road pricing are not part of the total travel costs, the upper bound of the efficiency loss depends on two parameters, namely $\gamma_{1}(\boldsymbol{C}, \boldsymbol{\tau})$ and $\gamma_{2}(\boldsymbol{C}, \boldsymbol{\tau})$. The upper bound of $\rho$ is a monotonically increasing function of $\gamma_{1}(\boldsymbol{C}, \boldsymbol{\tau})$ and $\gamma_{2}(\boldsymbol{C}, \boldsymbol{\tau})$. Parameters $\gamma_{1}(\boldsymbol{C}, \boldsymbol{\tau})$ and $\gamma_{2}(\boldsymbol{C}, \boldsymbol{\tau})$ are dimensionless number of the efficiency loss defined exclusively by the class of link travel cost functions and the road pricing scheme. When the road pricing scheme $\boldsymbol{\tau}$ is a zero vector, $\gamma_{2}(\boldsymbol{C}, \boldsymbol{\tau})$ is zero and the parameter $\gamma_{1}(\boldsymbol{C}, \boldsymbol{\tau})$ only depend on the link travel cost function, then $\rho \leq \frac{1}{1 \gamma,{ }_{1}(C \tau)}$.

\section{CONCLUSIONS}

In this paper, we have investigated the efficiency loss of UE-CN mixed traffic assignment under road pricing by analytic derivation. Firstly, we obtained the VI model of UE-
$\mathrm{CN}$ mixed traffic assignment under road pricing. Then, we derived the upper bound when the road pricing are (are not) part of the total travel costs. It is shown that the upper bound is a is a constant when road pricing is considered as part of total travel costs and the link travel cost functions satisfy some given conditions. The upper bound depends on the class of link travel cost functions and the road pricing scheme when road pricing is not considered as part of total travel costs. Our on-going study is to explore the tight lower upper bound and numerical results of the UE-CN mixed traffic assignment under road pricing.

\section{ACKNOWLEDGMENT}

This research was partly funded by the National Natural Science Foundation of China (71161005), the "125" Science and Technology Grand Project Sponsored by the Department of Education of Guizhou Province ([2012]011).

\section{REFERENCES}

[1] H. Yang, and H. J.Huang, Mathematical and Economic Theory of Road Pricing, Elsevier, Oxford,2005.

[2] T. Roughgarden, and E.Trados, “How bad is selfish routing?” Journal of the ACM , vol. 49, pp. 236-259, 2002.

[3] G. Karakostas, and S. G. Kolliopoulos, "The efficiency of optimal taxes." Combinatorial and Algorithmic Aspects of Networking, Lecture Notes in Computer Science, vol. 3405,pp. 3-12, 2005.

[4] H. Yang, W. Xu, and B. Heydecker, "Bounding the efficiency of road pricing.” Transportation Research Part E, vol. 46, pp. 90-108, 2010.

[5] D. R. Han, and H. Yang, "The multi-class, multi-criterion traffic equilibrium and the efficiency of congestion pricing." Transportation Research Part E, vol. 44, pp. 753-773,2008.

[6] X. J. Yu, and H. J. Huang, "Bounding efficiency loss of multiclass stochastic user equilibrium traffic assignment under road pricing." Journal of Jilin University (Engineering and Technology edition), vol. 39, pp. 71-75, 2009.

[7] K. D. Chen, D. L. Zhu, Y. H. Hu, and J. L .Liu, "The bound of price of anarchy for multiclass and multicriteria traffic equilibrium problem." Journal of Systems Science and Systems Engineering, vol. 21, pp. 77-93, 2012.

[8] D. R. Han and X. M. Yuan, "Existence of anonymous link tolls for decentralizing an oligopolistic game and the efficiency analysis." Journal of Industrial and Management Optimization, vol. 7, pp. 347364,2011

[9] T. L. Liu, J. Chen, and H. J. Huang, "Existence and efficiency of oligopoly equilbrium under toll and capacity comeptition." Transportation Research Part E, vol. 47, pp. 908-919, 2011.

[10] P. T. Harker, "Multiple equilibrium behaviors on networks." Transportation Science,vol. 22, pp. 39-46,1988.

[11] H. Yang, X. N. Zhang, and Q. Meng, "Stackelberg game and multiple equilibrium behaviors on network.” Transportation Research Part B, vol. 41, pp. 841-861, 2007.

[12] H. Yang, and X. N. Zhang, "Existence of anonymous link tolls for system optimum on networks with mixed equilibrium behaviors." Transportation Research Part B, vol. 42, pp. 99-112, 2008.

[13] X. J. Yu, and H. J. Huang, "Efficiency loss of mixed equilibrium behaviors with polynomial cost functions." Promet Traffic \& Transportation,vol. 22, pp. 325-331,2010.

[14] D. Kinderlehrer, and G. Stampacchia, An Introduction to Variational Inequalities and Their Applications, Academic Press, New York, 1986 